\title{
Identification of dielectric relaxations: a suitable infinite-dimensional state-space approach
}

\author{
P. Bidan ${ }^{1}$, L. Laudebat ${ }^{1^{*}}$, G. Montseny ${ }^{2}$ \\ ${ }^{1}$ Université Paul Sabatier, Laboratoire de Génie Electrique, \\ 118, route de Narbonne, 31062 Toulouse, France \\ ${ }^{2}$ Laboratoire d'Analyse et d'Architecture des Systèmes, \\ 7, avenue du Colonel Roche, 31077 Toulouse, France \\ * E-mail : laudebat@1get.ups-tlse.fr
}

\begin{abstract}
The concept of Diffusive Representation is used in this paper for modeling purposes in the field of electrical engineering, namely when some non-rational dynamics are involved [2]. Diffusive formulations are convenient for analysis and cheap numerical approximations. We study the problem of time-domain identification of the so-called diffusive symbol $\eta(\xi)$ associated to the permittivity $\varepsilon(\mathrm{p})$ of dielectric material. The so- obtained state-space models allow simple and precise time-domain simulation of such dynamical components.
\end{abstract}

\section{INTRODUCTION}

From an input-output point of view, the dipolar polarization of a large number of materials can be described by empirical models (Cole-Cole, Cole-Davidson etc.). On the basis of experimental observations, such models in general involve a non rational complex permittivity $\varepsilon(j \omega)$ [1] : In Curie-Von Schweidler behavior for example, polarization current follows a power-law time dependence $\mathrm{t}^{-\mathrm{n}} \quad(\mathrm{n}<1)$, associated to dielectric viscosity. When the involved dynamic operators are of rational type, classical state space formulations are efficiently and widely used in commercial simulators (Matlab-Simulink, Pspice etc.). In the non rational case however, the dimension of such formulations becomes necessarily infinite and so, accurate finite-dimensional approximations must be performed: this is the topic of the present paper.

From a synthetic point of view, state-space input-output models are linked to the input-output transfer function $\mathrm{H}(\mathrm{p})$ following:

$$
\begin{gathered}
\left\{\begin{array}{l}
\dot{X}(\mathrm{t})=A X(t)+B u(t) \\
y(t)=C X(t)+D u(t)
\end{array}\right. \\
\Downarrow \\
H(p)=\frac{Y(p)}{U(p)}=C(p I-A)^{-1} B+D ;
\end{gathered}
$$

$\mathrm{X}(\mathrm{t})$ finite-dimensional $\Rightarrow \mathrm{H}(\mathrm{p})$ rational $\mathrm{H}(\mathrm{p})$ non rational, $\Rightarrow \mathrm{X}(\mathrm{t})$ infinite-dimensional.

Diffusive representation is a convenient and efficient approach to the problem of state-space realization (1). The concept is presented in [3] in a general framework. In the simplified version used in this paper, the diffusive realization of a transfer function $\mathrm{H}(\mathrm{p})$ is the following input-output state-space representation:

$$
\left\{\begin{array}{rl}
\partial_{\mathrm{t}} \psi(\xi, \mathrm{t}) & =-\xi \psi(\xi, \mathrm{t})+\mathrm{u}(\mathrm{t}), \psi(0, \mathrm{t})=0, \xi \in \mathbb{R}^{+} \\
\mathrm{y}(\mathrm{t}) & =\int_{0}^{+\infty} \eta(\xi) \psi(\xi, \mathrm{t}) \mathrm{d} \xi
\end{array} .\right.
$$

where the so-called "diffusive symbol" ${ }^{1 "} \eta(\xi)$ is solution of the following integral equation (in general in the sense of distributions):

\begin{tabular}{|c|c|c|c|}
\hline & Permittivity $\varepsilon(\mathrm{p})$ & Diffusif Symbol $\eta(\xi)$ & Limitations \\
\hline Fraction & $\frac{1}{\mathrm{p}^{\alpha}}$ & $\frac{\sin \alpha \pi}{\pi} \frac{1}{\xi^{\alpha}}$ & $0<\alpha<1$ \\
\hline Debye & $\frac{1}{1+\tau_{0} \mathrm{p}}$ & $\xi_{0} \delta\left(\xi-\xi_{0}\right)$ & $\xi_{0}=1 / \tau_{0}$ \\
\hline Cole-Cole & $\frac{1}{1+\left(\tau_{0} \mathrm{p}\right)^{\alpha}}$ & $\frac{\frac{\sin (\alpha \pi)}{\pi}}{\left(\frac{\xi}{\xi_{0}}\right)^{\alpha}+2 \cos (\alpha \pi)+\left(\frac{\xi_{0}}{\xi}\right)^{\alpha}}$ & $0<\alpha<1$ \\
\hline Cole-Davidson & $\frac{1}{\left(1+\tau_{0} \mathrm{p}\right)^{\beta}}$ & $\frac{\sin \alpha \pi}{\pi} \frac{1}{\left(\xi-\xi_{0}\right)^{\beta}}$ & $\begin{array}{c}\xi>\xi_{0} \\
0<\beta<1\end{array}$ \\
\hline Havriliak-Negami & $\frac{1}{\left(1+\left(\tau_{0} \mathrm{p}\right)^{\alpha}\right)^{\beta}}$ & $\frac{\left.\left.\left[\frac{\xi_{0}}{\xi}\right)^{\alpha}+\mathrm{e}^{\mathrm{j} \alpha \pi}\right]^{\beta}-\left[\frac{\xi_{0}}{\xi}\right)^{\alpha}+\mathrm{e}^{-j \alpha \pi}\right]^{\beta}}{\left.2 j \pi\left[\frac{\xi_{0}}{\xi}\right)^{\alpha}+2 \cos (\alpha \pi)+\left(\frac{\xi}{\xi_{0}}\right]^{\alpha}\right]^{\beta}}$ & $\begin{array}{c}0<\alpha<1 \\
0<\beta<1\end{array}$ \\
\hline
\end{tabular}

Table 1 : Diffusive symbol of dielectric models

\footnotetext{
${ }^{1}$ This term is justified by mathematical reasons presented in [3],[4].
} 


$$
\mathrm{H}(\mathrm{j} \omega)=\int_{0}^{+\infty} \frac{\eta(\xi)}{\mathrm{j} \omega+\xi} \mathrm{d} \xi \omega \in \mathbb{R}
$$

Denoting by $h(t)$ the impulse response associated to $H(p)$, the diffusive symbol $\eta$ is also given by :

$$
\eta=\mathcal{L}^{-1} h \text {, }
$$

which allows concrete analytical computations of $\eta$ by use of Laplace transform (compare with $\mathrm{H}(\mathrm{p})=\mathcal{L} \mathrm{h}$ ).

\section{MODELING OF DIELECTRIC MATERIALS}

Some classical empirical models based on frequency behaviors (Cole-Cole, Cole-Davidson, etc.) are given in Table 1. In figure 1 we can see the graphs of the associated diffusive symbols [11].

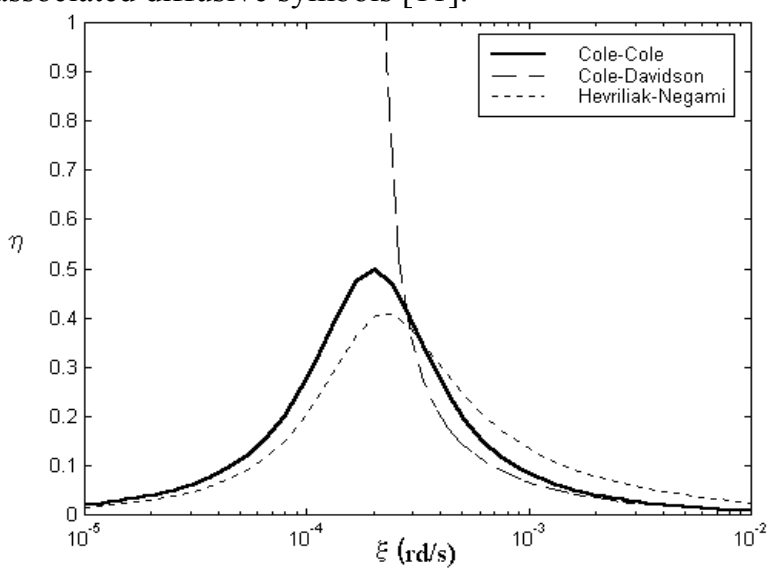

Figure 1 : Diffusive symbols

In this context, both relationships are equivalent:

$$
\begin{gathered}
\mathrm{D}(\mathrm{p})=\varepsilon_{0}\left(\varepsilon_{\mathrm{s}}-\varepsilon_{\infty}\right) \varepsilon(\mathrm{p}) \mathrm{E}(\mathrm{p}) \\
\mathbb{\Downarrow} \\
\left\{\begin{array}{c}
\partial_{\mathrm{t}} \psi(\xi, \mathrm{t})=-\xi \psi(\xi, \mathrm{t})+\mathrm{E}(\mathrm{t}), \quad \psi(\xi, \mathrm{t})=0, \quad \xi \in \mathbb{R}^{+} \\
\mathrm{D}(\mathrm{t})=\varepsilon_{0}\left(\varepsilon_{\mathrm{s}}-\varepsilon_{\infty}\right) \int_{0}^{+\infty} \eta(\xi, \mathrm{t}) \psi(\xi, \mathrm{t}) \mathrm{d} \xi
\end{array}\right.
\end{gathered}
$$

where E and D denote respectively the electrical and displacement fields. In an equivalent way, $\varepsilon(p)$ and $\eta(\xi)$ characterize the dielectric dynamics. The state-space representation (6), although infinite-dimensional, is easily approximated by discretization on the $\xi$ variable in order to obtain an implementable model. The state-space representation (6) can be viewed as an explicit exact dynamic model for the dielectric itself.

The practical determination of the discretization mesh $\left\{\xi_{\mathrm{n}}\right\}$ of course depends on the diffusive symbol $\eta(\xi)$. The boundaries are imposed by the dynamic properties of the system. Namely, slowest dynamics $\psi\left(\xi_{\min }, \mathrm{t}\right)$ are associated to long time behaviors and the fastest dynamics $\psi\left(\xi_{\max }, t\right)$ to short ones.

The approximated model is therefore given by [6]:

$$
\left\{\begin{array}{rl}
\frac{\mathrm{d}}{\mathrm{d}} \psi_{\mathrm{k}}(\mathrm{t}) & =-\xi_{\mathrm{k}} \psi_{\mathrm{k}}(\mathrm{t})+\mathrm{E}(\mathrm{t}), \psi_{\mathrm{k}}(0)=0, \mathrm{k}=1, \ldots, \mathrm{K} \\
\mathrm{D}(\mathrm{t}) & =\varepsilon_{0}\left(\varepsilon_{\mathrm{s}}-\varepsilon_{\infty}\right) \sum_{\mathrm{k}=1}^{\mathrm{K}} \eta_{\mathrm{k}} \psi_{\mathrm{k}}(\mathrm{t})
\end{array} .\right.
$$

As an example, the step response of ( 7 ) for $E=100 \mathrm{~V} / \mathrm{m}$ is shown in figure 2. The current density is associated to the dynamic polarization of the dipole relaxation following:

$$
J(t)=\frac{d}{d t} D(t)
$$

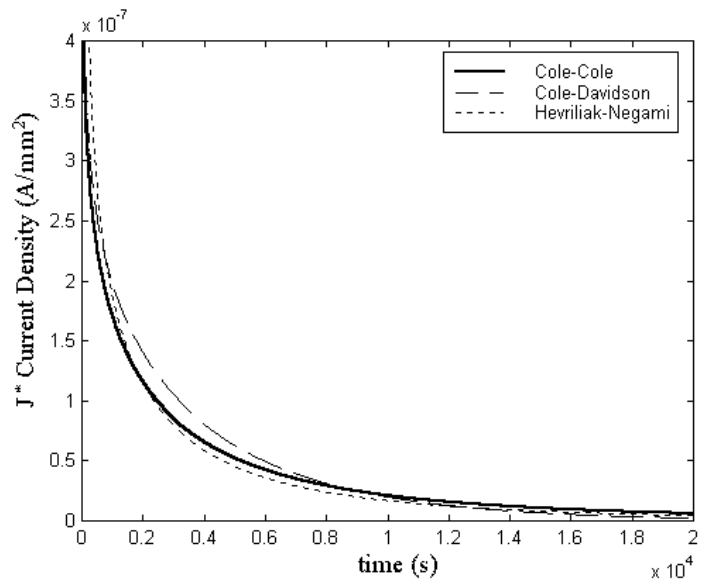

Figure 2 : Simulated density current with step electrical field $\mathrm{E}(\mathrm{t})$

\section{IDENTIFICATION OF THE DIFFUSIVE SYMBOL FROM PHYSICAL MEASUREMENTS}

\section{Introduction}

Identification is devoted to the determination of the diffusive symbol $\eta(\xi)$, which in fact summarizes the dynamic complexity of the system. Note that non linear problems can also be tackled following the diffusive approach (see [7]).

The identification problem can be treated in the frequency as well as time domains. We only present here the second one. The major interest of model (6) lies in the fact that the function $(\eta)$ to be identified appears linearly in the output expression. Furthermore, the diffusive nature of (7) leads to both accurate and cheap numerical approximations $[4,5]$.

\section{Principle}

From a practical point of view, the identification is realized from temporal measurements of the displacement field D, collected in a vector denoted by $\dddot{\mathbf{D}}$. The linear dependence of $\mathrm{D}$ with respect to $\eta$ can be expressed through a linear operator $\mathbf{A}_{\mathbf{E}}$, in fact a matrix in the case of the finite-dimensional approximation (7). Operator $\mathbf{A}_{\mathbf{E}}$ is depending on the input $\mathrm{E}(\mathrm{t})$ (and so is varying with respect to time); so we have :

$$
\mathbf{D}=\mathbf{A}_{\mathrm{E}} \eta \text {. }
$$

The (optimal) symbol $\hat{\eta}$ derived from (9) in the sense of 
minimizing the Euclidean distance $\|\mathbf{D}-\dddot{\mathbf{D}}\|^{2}$ is classically obtained by orthogonal projection. It is expressed by:

$$
\hat{\eta}=\mathbf{A}_{\mathbf{E}}+\dddot{\mathbf{D}}
$$

where $\mathbf{A}_{\mathrm{E}}{ }^{\star}$ is the standard pseudo-inverse of $\mathbf{A}_{\mathrm{E}}$, defined by $[8]^{2}$ :

$$
\mathbf{A}_{\mathrm{E}}{ }^{*}=\left(\mathbf{A}_{\mathrm{E}}{ }^{*} \mathbf{A}_{\mathrm{E}}\right)^{-1} \mathbf{A}_{\mathrm{E}}{ }^{*} \cdot
$$

Remark: A recursive solution using an optimal Kalman filter can also be constructed, allowing online identification as soon as measurements are available [9].

\section{Example}

We consider the standard experiment related to the polarization current in a plate capacitor; the dielectric is here the material under study. The measurement device consists in an electrometer with an external supply. The amount of charge $q(t)$ is determined from the measured current $\mathrm{i}(\mathrm{t})$ by:

$$
q(t)=\int_{0}^{t} i(\tau) d \tau,
$$

and the displacement field is given by:

$$
D(t)=\frac{q(t)}{S},
$$

where $\mathrm{S}$ is the surface of the electrode capacitor.

The material under study is an aluminium nitride ceramics AlN. It is an attractive substrate material currently used in high power packaging applications thanks to both its thermal and electrical properties.

The comparison between the measured current and the one obtained from the identified model is presented on figure 3 . The diffusive formulation clearly gives accurate results.

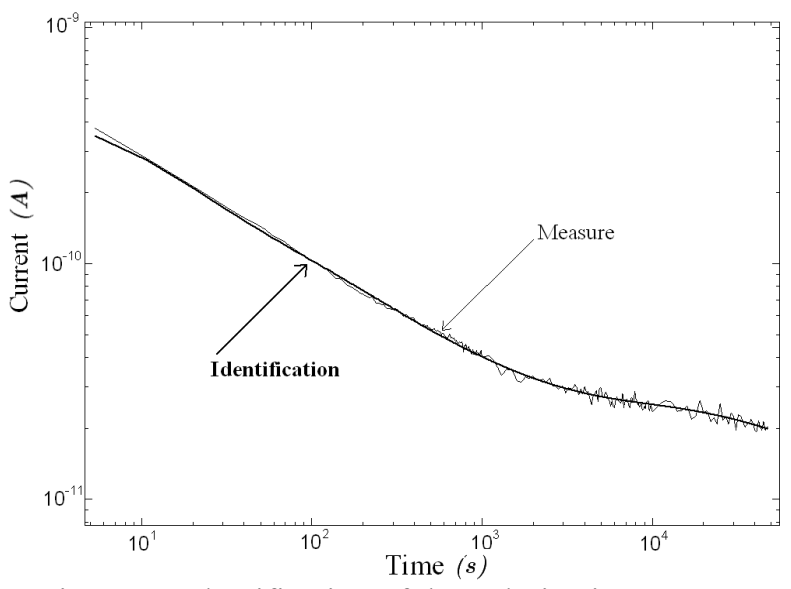

Figure 3 : Identification of the polarization current.

Around $\mathrm{t}=2.10^{3} \mathrm{~s}$, we can observe a break; this suggests that there are two processes involved: one which prevails

\footnotetext{
${ }^{2}$ As usual, ${ }^{*}$ denotes the transpose-conjugated of a complex vector
}

at long times, the other at short times. The identified diffusive symbol is shown in figure 4 . An enlargement of the zone in dotted line distinguishes the two supposed processes: a relaxation peak is clearly highlighted around $10^{-3} \mathrm{rad} / \mathrm{s}$.

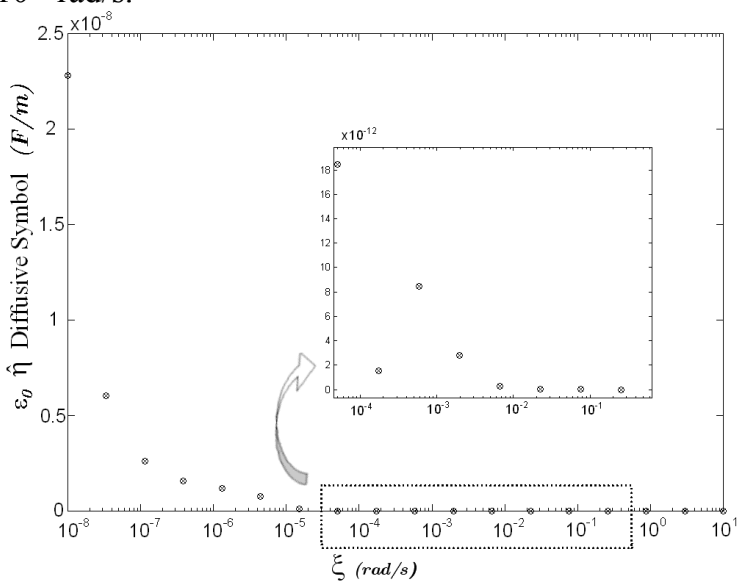

Figure 4 : Diffusive symbol of the AIN ceramic

\section{CONCLUSION}

Diffusive representation approach reveals to be at the same time flexible, convenient and efficient in modeling, numerical simulation and identification of complex dynamics. Time as well as frequency domains are concerned and dielectric models can therefore be included in complex simulation environments like cells commutation of power electronics devices for example. Cheap and precise approximations can be elaborated under suitable differential forms which must be viewed as convenient models useful for various applications [6], [11]: propagation lines, CAD, synthesis of distributed filters, thermo-electrical coupling, etc.

Further details will be found in [6,11], namely about energy dissipation properties of the diffusive formulation not presented here and which gives some physical meaning to the notion of diffusive symbol and realization.

\section{REFERENCES}

[1] A.K. Jonsher, Dielectric Relaxation in Solids, Chelsea Dielectrics Press, London 1983.

[2] P. Bidan, T. Lebey, G. Montseny, C. Neascu, J. Saint-Michel, "Transient voltage distribution in inverter fed motor winding : experimental study and modeling", IEEE Transactions on Power Electronics, Vol 16, No 1, January 2001.

[3] G. Montseny, Représentation diffusive, To appear.

[4] G. Monstseny, "Diffusive representation of pseudo-differential time-operators", ESAIM: Proc. vol 5, pp 159-175, December 1998,

[5] G. Montseny, "Diffusive representation: a new concept for complex dynamic problems involving pseudodifferential operators", Lecture notes of the Summer School "On the links between nonlinear physics 
and information sciences", Les Houches Center of Physics (France), Sept. 8-13, 2002.

[6] L. Laudebat, P. Bidan, G. Montseny "Modeling and optimal identification of pseudodifferential electrical dynamics by means of diffusive representation - Part I : Modeling", IEEE circuits and systems, to appear.

[7] J. Audounet, V. Giovangigli, J.-M. Roquejoffre, "A threshold phenomenon in the propagation of a point-source initiated flame", Physica D, pp 295-316, 1998.

[8] L. Ljung, "System Identification, theory for the user", Upper Saddle River, Prentice Hall, New Jersey, 1999.

[9] L. Laudebat, P. Bidan, "Quelques résultats d'iden-tification et poursuite sous représentation diffusive de modèles pseudo-différentiels en Génie Electrique": JDA'2001 Journée Doctorale d'Automatique Toulouse 25-27 septembre 2001.

[10] J. R. Mc Donald, "Impedance Spectroscopy: Emphazing Solid Materials and System", A Wiley-Interscience Publication, John Wiley \& Sons, 1987.

[11] L. Laudebat, "Modélisation et identification sous representation diffusive de comportements dynamiques non rationnels en genie électrique", PHD thesis, Toulouse University, 2003. 